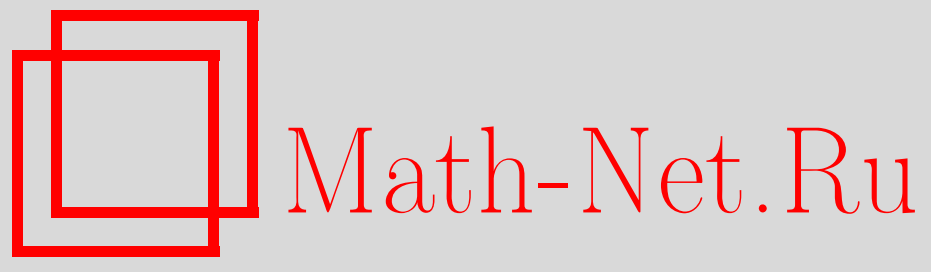

М. А. Браун, Начальное условие для эволюции пертурбативного КХД-померона в ядре, ТМФ, 2006, том 148, номер 1, 57-63

DOI: https://doi.org/10.4213/tmf2057

Использование Общероссийского математического портала Math-Net.Ru подразумевает, что вы прочитали и согласны с пользовательским соглашением http://www . mathnet.ru/rus/agreement

Параметры загрузки:

IP: 54.147 .182 .235

26 апреля 2023 г., 16:11:53

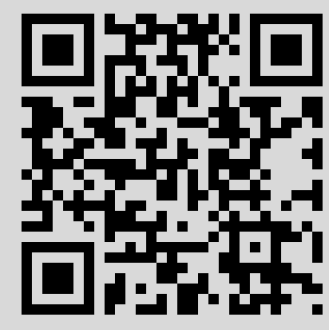




\title{
НАЧАЛЬНОЕ УСЛОВИЕ ДЛЯ ЭВОЛЮЦИИ ПЕРТУРБАТИВНОГО КХД-ПОМЕРОНА В ЯДРЕ
}

\begin{abstract}
Показано, что субдоминантные члены, полученные с помощью реджезованной глюонной диаграммной техники, которые следует добавить к померонным веерным диаграммам с трехпомеронным взаимодействием, можно точно учесть, если выбрать начальное условие эволюции в глауберовом виде. Это демонстрирует полную эквивалентность дипольной картины и реджезованной глюонной техники в высокоэнергетическом пределе не только в ведущем порядке, но также и в следующем порядке.
\end{abstract}

Ключевые слова: реджезованные глюоны, КХД-померон, дипольная картина, высокие энергии, взаимодействие с ядром.

\section{1. ВВЕДЕНИЕ}

Как показано в работах [1], уравнение распространения пертурбативного КХДпомерона в ядре (уравнение Балицкого-Ковчегова (БК) [2], [3]) соответствует суммированию веерных диаграмм, построенных из функций Грина померона, связанных расщепляющейся трехпомеронной вершиной $V$, впервые введенной в дипольной картине в работе [4], а затем полученной для произвольного числа цветов из реджезованных глюоных диаграмм в работе [5] и упрощенной в случае большого числа цветов в работе [6]. В последнем подходе, однако, вклад трехпомеронного взаимодействия не исчерпывает полную амплитуду. Кроме того, появляется вклад от так называемого "редуцированого" или "реджезованного" члена, который дается однопомеронным обменом, взаимодействующим со всеми цветными диполями в многонуклонной мишени. Этот вклад был явно введен для четырех глюонов в работе [5] и для шести глюонов в работе [7]. Реджезованный вклад, очевидно, является субдоминантным при высоких энергиях, поэтому его учет в уравнении БК на самом деле не оправдан. Однако уже на ранней стадии обсуждения уравнения БК мы предположили, что можно воспроизвести реджезованный вклад с помощью подходящего обобщения начального условия для эволюции. На эту идею нас натолкнуло обсуждение с Ю. Ковчеговым и Е. Левиным начального условия типа условия

* Санкт-Петербургский государственный университет, Санкт-Петербург, Россия. E-mail: braun1@pbox.spbu.ru 
Глаубера, предложенного в работе [3]. В частности, Е. Левин верно заметил, что множественные двухглюонные обмены не содержатся в диаграммах трехпомеронного взаимодействия и должны быть добавлены путем глауберизации однонуклонного начального условия [8]. Строго говоря, этот факт не является важным ни для формулировки уравнения БК, ни для его решения, поскольку относится к субдоминантным вкладам и, как показывают вычисления, практически перестает влиять на решение уже после эволюции на несколько единиц быстроты. Однако интересно выяснить, можно ли распространить соответствие между дипольной картиной и реджезованной глюонной техникой также и на субдоминантный уровнь. Некоторое практическое значение может также иметь исследование эволюции до не столь высоких быстрот, когда результат сохраняет некоторый след выбранного начального условия.

В настоящей работе мы показываем, что реджезованные члены, найденные с помощью реджезованной глюонной техники, при добавлении к членам трехпомеронного взаимодействия действительно точно воспроизводятся за счет выбора начального условия типа условия Глаубера в уравнении БК.

\section{2. НАЧАЛЬНОЕ УСЛОВИЕ ЭВОЛЮЦИИ}

Амплитуду рассеяния на ядре для некоторой налетающей частицы с быстротой $y$ и фиксированным прицельным параметром $b$ можно представить в виде

$$
\mathcal{A}(y, b)=2 i s \int d^{2} r \rho(r) \Phi(r, y, b)
$$

где $\rho(r)$ - цветовая дипольная плотность в налетающей частице, а $\Phi(r, y, b)$ - амплитуда рассеяния диполя на ядре. В реджезованной глюонной диаграммной технике она имеет вид суммы всех веерных диаграмм, построенных из БФКЛ-функций Грина $^{1)} G$ и трехпомеронной вершины $V$ для расщепления померона на два. Для наших целей их явный вид нам не нужен, его можно найти в цитированной литературе. Нам потребуется член низшего порядка, соответствующий однопомеронному обмену:

$$
\Phi_{1}(r, y, b)=\int d^{2} r^{\prime} G\left(y, r, r^{\prime}\right) A T(b) \rho_{N}\left(r^{\prime}\right)
$$

где $G\left(y, r, r^{\prime}\right)$ - БФКЛ-функция Грина (для рассеяния вперед), $A T(b)$ - поперечная ядерная плотность для прицельного параметра $b$, а $\rho_{N}(r)$ - цветовая дипольная плотность нуклона.

Для сравнения с результатами, полученными с помощью реджезованной глюоной диаграммной техники, введем померон, взаимодействующий с налетающей частицей:

$$
P(y, r)=\int d^{2} r^{\prime} \rho\left(r^{\prime}\right) G\left(y, r^{\prime}, r\right)
$$

\footnotetext{
1) Мы имеем в виду функции Грина, рассмотренные в работах Балицкого, Фадина, Кураева, Липатова.
} 
и представим редуцированную амплитуду $\mathcal{A} /(2 i s)$ в виде

$$
\tilde{\mathcal{A}}(y, b) \equiv \int d^{2} r \rho(r) \Phi(r, y, b)=\int d^{2} r P(y, r) A T(b) \rho_{N}(r) .
$$

Затем ампутируем померон $P(r)$ со стороны мишени, т.е. выделим глюонные пропагаторы $1 / k^{4}$ из его фурье-преобразования, включив их в прицельный множитель мишени, и запишем выражение (4) в виде

$$
\tilde{\mathcal{A}}(y, b)=\int d^{2} r D(y, r) \tau(r, b) .
$$

Здесь $D(y, r)$ - ампутированный померон, взаимодействующий с налетающей частицей, а $\tau(r, b)$ определяется как преобразование Фурье:

$$
\tau(r, b)=A T(b) \int \frac{d^{2} k}{(2 \pi)^{2} k^{4}} e^{i k r} \rho_{N}(k) .
$$

На первый взгляд выражение (6) инфракрасно сингулярно, поскольку интеграл в нем расходится при $k \rightarrow 0$. Однако эта расходимость является нефизической. В действительности в выражении (6) можно вычесть единицу из экспоненты, представив $\tau(r)$ в виде

$$
\tau(r, b)=\tau(0, b)+\tilde{\tau}(r, b)
$$

где функция

$$
\tilde{\tau}(r)=\int \frac{d^{2} k}{(2 \pi)^{2} k^{4}}\left(e^{i k r}-1\right) \rho_{N}(k)
$$

является инфракрасно конечной, а $\tau(0)$ - инфракрасно расходящейся, но не зависящей от $r$. Поскольку в выражении (5)

$$
\int d^{2} r D(y, r)=0
$$

можно опустить первое слагаемое в выражении (7) и записать

$$
\tilde{\mathcal{A}}(y, b)=g^{2} \int d^{2} r \widetilde{D}(r, y) \tilde{\tau}(r, b),
$$

где мы также выделили $g^{2}$ из $D$, положив

$$
D(r, y)=g^{2} \widetilde{D}(r, y)
$$

Выражение (10) является, очевидно, инфракрасно конечным.

Наша следующая цель состоит в том, чтобы посмотреть, можно ли, изменив подходящим образом в (10) начальное условие, задаваемое прицельным множителем $g^{2} \tilde{\tau}(r)$, воспроизвести реджезованные члены, найденные при анализе реджезованных глюонных диаграмм. Аргументы $y$ и $b$ далее всегда остаются фиксированными, и мы их вообще не указываем. 


\section{3. ДВА ЦЕНТРА РАССЕЯНИЯ}

Начнем со вклада от обмена четырех реджезованных глюонов $D_{4}$, который был подробно исследован в работах [5], [6], [9]. Было получено, что полный вклад можно разделить на два слагаемых: слагаемое, соответствующее трехпомеронному взаимодействию, и реджезованное слагаемое $D_{4}^{(\mathrm{R})}$, соответствующее однопомеронному обмену при взаимодействии с обоими центрами мишени. Естественно, при асимптотических энергиях реджезованный член является субдоминантным, поскольку если $\Delta$ является БФКЛ-интерсептом (в действительности - померонным интерсептом минус единица), то $D_{4}^{(\mathrm{R})}$ ведет себя как $\exp (\Delta y)$, в то время как трехпомеронный член ведет себя как $\exp (2 \Delta y)$.

Вклад реджезованного члена в амплитуду имеет вид

$$
\tilde{\mathcal{A}}_{4}^{(\mathrm{R})}=\frac{1}{2} \int d^{2} r_{1} d^{2} r_{2} D_{4}^{(\mathrm{R})}\left(r_{1}, r_{2}\right) \tau\left(r_{1}\right) \tau\left(r_{2}\right)
$$

где $D^{(\mathrm{R})}\left(r_{1}, r_{2}\right)$ - фурье-преобразование реджезованной части для двух рассеянных вперед померонов:

$$
D_{4}^{(\mathrm{R})}\left(r_{1}, r_{2}\right)=\int \frac{d^{2} k_{1}}{(2 \pi)^{2}} \frac{d^{2} k_{2}}{(2 \pi)^{2}} e^{-i k_{1} r_{1}-i k_{2} r_{2}} D_{4}^{(\mathrm{R})}\left(k_{1},-k_{1}, k_{2},-k_{2}\right) .
$$

Здесь $D_{4}^{(\mathrm{R})}\left(k_{1}, k_{2}, k_{3}, k_{4}\right)$ - реджезованный член как функция глюонных импульсов в пределе большого количества цветов (см. [5], [6]):

$$
D_{4}^{(\mathrm{R})}(1,2,3,4)=\frac{1}{2} g^{2}\left(\sum_{i=1}^{4} D(i)-\sum_{i=2}^{4} D(1 i)\right)
$$

в сокращенных обозначениях $k_{1} \equiv 1, k_{1}+k_{2} \equiv 12$ и т.д. В нашем случае рассеяния вперед, учитывая, что $D(0)=0$, имеем

$$
D_{4}^{(\mathrm{R})}\left(k_{1},-k_{1}, k_{2},-k_{2}\right)=\frac{1}{2} g^{2}\left(2 D\left(k_{1}\right)+2 D\left(k_{2}\right)-D\left(k_{1}+k_{2}\right)-D\left(k_{1}-k_{2}\right)\right) .
$$

Применяя преобразование Фурье, находим

$D_{4}^{(\mathrm{R})}\left(r_{1}, r_{2}\right)=\frac{1}{2} g^{2}\left(2 \delta^{2}\left(r_{2}\right) D\left(r_{1}\right)+2 \delta^{2}\left(r_{1}\right) D\left(r_{2}\right)-g^{2} \delta^{2}\left(r_{1}+r_{2}\right) D\left(r_{1}\right)-g^{2} \delta^{2}\left(r_{1}-r_{2}\right) D\left(r_{1}\right)\right)$.

Подставляя этот результат в выражение (12), получаем

$$
\tilde{\mathcal{A}}_{4}^{(\mathrm{R})}=\frac{1}{2} g^{2}\left\{2 \tau(0) \int d^{2} r D(r) \tau(r)-\int d^{2} r D(r) \tau^{2}(r)\right\},
$$

или, если представить $\tau(r)$ и $D(r)$ в соответствии с выражениями (7) и (11),

$$
\tilde{\mathcal{A}}_{4}^{(\mathrm{R})}=-\frac{1}{2} g^{4}\left\{\int d^{2} r \widetilde{D}(r) \tilde{\tau}^{2}(r)+\tau^{2}(0) \int d^{2} r \widetilde{D}(r)\right\} .
$$


Второе слагаемое равно нулю, так что окончательно вклад реджезованного члена в амплитуду имеет вид

$$
\tilde{\mathcal{A}}_{4}^{(\mathrm{R})}=\int d^{2} r \widetilde{D}(r)\left[-\frac{1}{2}\left(g^{2} \tilde{\tau}(r)\right)^{2}\right] .
$$

Сравнивая полученное выражение с выражением (10), мы видим, что этот вклад воспроизводится, если в члене низшего порядка совершить подстановку

$$
g^{2} \tilde{\tau}(r) \rightarrow g^{2} \tilde{\tau}(r)-\frac{1}{2}\left(g^{2} \tilde{\tau}(r)\right)^{2},
$$

что в точности соответствует глауберизации начального условия

$$
g^{2} \tilde{\tau}(r) \rightarrow 1-e^{-g^{2} \tilde{\tau}(r)}
$$

\section{4. ПРОИЗВОЛЬНОЕ ЧИСЛО ЦЕНТРОВ РАССЕЯНИЯ}

Приведенный вывод легко обобщить на произвольное (четное) число глюонов $n=2 p$. Используя выражение для вершины, связывающей виртуальный фотон с $n$ глюонами, разбитыми на бесцветные пары $\{12\},\{34\}, \ldots,\{(n-1) n\}$, найденное в пределе $N_{c} \rightarrow \infty$ в работе [10], получаем в этом пределе

$$
D_{n}^{(\mathrm{R})}\left(k_{1}, \ldots, k_{n}\right)=-\frac{g^{n-2}}{2^{p}} \int d^{2} r D(r) \prod_{i=1}^{n}\left(e^{i k_{i} r}-1\right) .
$$

Можно проверить, что это выражение совпадает с (14) при $n=4$, если использовать функцию $D_{6}^{(\mathrm{R})}$, полученную в работе [7] для общего $N_{c}$, при $N_{c} \rightarrow \infty$. В нашем случае мы должны взять каждую мишень с нулевым передаваемым импульсом, т.е. положить $k_{1}+k_{2}=k_{3}+k_{4}=\cdots=k_{n-1}+k_{n}=0$, и с $p$ импульсами $k_{1}, k_{3}, \ldots, k_{n-1}$ в качестве независимых переменных. Чтобы ввести взаимодействие с мишенью, преобразуем выражение $(22)$ к координатному пространству $r_{1}, r_{3}, \ldots, r_{n-1}$ :

$$
\begin{aligned}
& D_{n}^{(\mathrm{R})}\left(r_{1}, r_{3}, \ldots, r_{n-1}\right)= \\
& \quad=-\frac{g^{n-2}}{2^{p}} \int d^{2} r D(r) \prod_{i=1}^{p}\left[\frac{d^{2} k_{2 i-1} e^{i r_{2 i-1} k_{2 i-1}}}{(2 \pi)^{2}}\left(2-e^{i k_{2 i-1} r}-e^{-i k_{2 i-1} r}\right)\right] .
\end{aligned}
$$

Вклады в интеграл, которые вносят постоянные слагаемые в одном или нескольких множителях в круглых скобках, дают члены, содержащие $\delta^{2}\left(r_{i}\right)$ или несколько таких множителей. После интегрирования с отвечающими мишени множителями $\tau\left(r_{i}\right)$ они дают инфракрасно расходящиеся члены, содержащие $\tau(0)$. Однако мы с самого начала знали, что такие члены должны сокращаться, поскольку исходное выражение (22) очевидным образом интегрируемо с отвечающим мишени множителем в инфракрасной области ${ }^{2)}$. Зная это, мы можем опустить двойки в выражении (23). Более того, очевидно, что оба члена, остающиеся в скобках, дают одинаковый вклад после интегрирования с отвечающим мишени множителем, поскольку

\footnotetext{
2) Мы признательны Дж. П. Вакке за то, что он обратил наше внимание на этот факт.
} 
замена $\mathbf{r} \rightarrow-\mathbf{r}$ не изменяет величины вклада. Таким образом, мы получаем $2^{p}$ одинаковых членов и легко находим окончательный результат в виде

$$
D_{n}^{(\mathrm{R})}\left(r_{1}, r_{3}, \ldots, r_{n-1}\right)=-(-1)^{p} g^{n-2} D\left(r_{1}\right) \prod_{i=2}^{p-1} \delta^{2}\left(r_{2 i-1}-r_{1}\right)
$$

(при $n=2$ нет $\delta$-функций и $D_{2}^{(\mathrm{R})}(r)=D(r)$, как и ожидалось). Интегрируя это выражение с $p$ множителями, отвечающими мишени, с учетом симметрийного множителя $1 / p$ ! получаем

$$
\tilde{\mathcal{A}}_{n}^{(\mathrm{R})}=-(-1)^{p} \frac{1}{p !} g^{2 p-2} \int d^{2} r D(r) \tau^{p}(r)
$$

Исключая инфракрасно расходящиеся вклады от степеней $\tau(0)$, которые, как было отмечено, должны полностью сокращаться, и переходя к $\widetilde{D}$, окончательно находим

$$
\tilde{\mathcal{A}}_{2 p}^{(\mathrm{R})}=-(-1)^{p} \frac{1}{p !} g^{2 p} \int d^{2} r \widetilde{D}(r) \tilde{\tau}^{p}(r)
$$

Суммируя вклады для всех $p$, получаем глауберово выражение под знаком интеграла по $r$ :

$$
\tilde{\mathcal{A}}^{(\mathrm{R})}=\sum_{p=1} \tilde{\mathcal{A}}_{2 p}^{(\mathrm{R})}=\int d^{2} r \widetilde{D}(r)\left(1-e^{-g^{2} \tilde{\tau}(r)}\right) .
$$

Это означает, что все реджезованные вклады действительно можно адекватно учитывать с помощью глауберизации начального условия в соответствии с выражением (20).

\section{5. ЗАКЛЮЧЕНИЕ}

Мы показали, что субдоминантные члены, полученные с помощью реджезованной глюонной диаграммной техники, которые следует добавить к померонным веерным диаграммам с трехпомеронным взаимодействием, можно точно учесть, если выбрать начальное условие эволюции в глауберовом виде. Это демонстрирует полную эквивалентность дипольной картины и реджезованной глюонной техники не только в ведущем порядке в высокоэнергетическом пределе, но также и в следующем порядке.

Как было отмечено, глауберизация начального условия не оказывает никакого влияния на решение после эволюции на несколько единиц быстроты: уравнение БК быстро “забывает" подробности начального условия. Однако на более ранних этапах эволюции это влияние, конечно, ощутимо; этот факт может иметь важное значение для практических применений.

Благодарности. Автору помогли многочисленные обсуждения с Й. Бартельсом, Ю. Ковчеговым, Е. Левиным и Дж. П. Ваккой. Данная работа выполнена при поддержке НАTO (грант PST.CLG.980287). 


\section{Список литературы}

[1] M. A. Braun, Eur. Phys. J. C, 16 (2000), 337; J. Bartels, L. N. Lipatov, G. P. Vacca, Nucl. Phys. B, 706 (2005), 391.

[2] I. Balitsky, Nucl. Phys. B, 463 (1996), 99.

[3] Yu. Kovchegov, Phys. Rev. D, 60 (1999), 034008.

[4] A. Mueller, B. Patel, Nucl. Phys. B, 425 (1994), 471.

[5] J. Bartels, M. Wuesthoff, Z. Phys. C, 66 (1995), 157.

[6] M. A. Braun, G. P. Vacca, Eur. Phys. J. C, 6 (1999), 147.

[7] J. Bartels, C. Ewerz, JHEP, 9909 (1999), 026.

[8] Е. Левин, частное сообщение.

[9] M. A. Braun, Eur. Phys. J. C, 6 (1999), 321.

[10] M. A. Braun, Z. Phys. C, 71 (1996), 601.

Поступила в редакцию 7.11.2005 